\title{
Research of Single Unit Fuzzy Event Cognitive Computing Based on Goal .
}

\author{
Kang Feng \\ School of Computer, Huainan Normal University \\ Huainan 232038, China \\ 15855432472@163.com
}

\begin{abstract}
To find the expressions of cognition, a single unit fuzzy event cognitive computing based on goal was designed. The cognitive data were single unit fuzzy events, cognition, inputting cognition, goals, works, methods and problems; the performance was measured in cognitive computation performance figure. The experimental results show that the single unit fuzzy event cognitive computing based on goal corrects the shortage of the existing cognitive computing and it has some key features, that the work achievement rate, the problem repeating rate and the rejected rate are ideal while the forgetting rate is very small, but they are bad while the forgetting rate is very large, the features accords with the cognitive laws. Thus cognition is able to be expressed with the single unit fuzzy event cognitive computing based on goal.
\end{abstract}

Keywords—cognitive computation; brain models; cognition; memory architecture; cognitive systems

\section{Introduction}

Cognition is the complete process of observing the world, understanding the world and reforming the world by the goal, as showed in figure 1 .

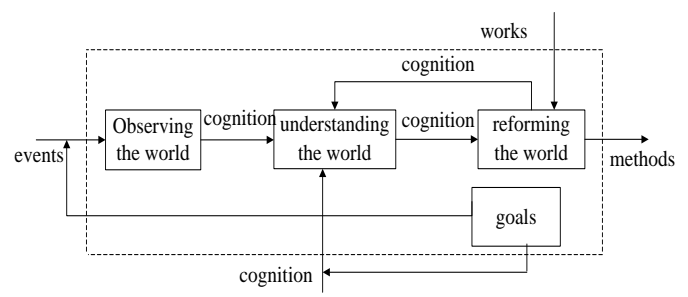

Fig.1 Cognition

*Anhui Provincial Natural Science Foundation (1508085MF124), the National Natural Science Foundation of China (11401243).

\author{
Xuemei Shi \\ School of Computer, Huainan Normal University \\ Huainan 232038, China \\ 2642309232@qq.com
}

In Fig.1, events, goals, works and methods were called cognitive data, so cognition was the processing of all cognitive data[1]. Relating the definition which "computing" was the processing of information, so each processing of single cognitive data was cognitive sub-computing and the processing of all cognitive data was cognitive computing. Cognitive computing was scientific means that expressed cognition, found the law of cognition, and explored the mysteries of the mankind mind [2].

II. The existing Cognitive computing research

Symbol Cognitive computing was the earliest cognitive computing, it was the processing of physical symbol, the cognitive data was a set of precise physical symbol, the representative figure in cognitive computing of symbols was Allen Newell, who put forward SOAR(State of the Operator And Results) cognitive computing[3], and John $\mathrm{K}$ Anderson who proposed ACT (the Adaptive Control of Thought) cognitive computing[4]. Connectionism cognitive computing was the simulation of real neural networks, so it was also known as artificial neural network. Hebbian learning rules, Perceptron learning rule and Delta learning rule were the general learning rules[5]. Biomedical signal cognitive computing directly detected the cognitive activities of the brain and the nervous system, it changed the physiological indexes of the cognitive activity into digital signals, those digital signals were the cognitive data. The biomedical technology was ERP (Event Related 
Potential), PET (Positron Emission Tomography), fMRI (Functional Magnetic Resonance Imaging) [6].

In the existing cognitive computing, the cognitive data didn't include all cognitive data , the existing cognitive computing didn't simulate cognition and the existing cognitive computing didn’t preprocess inputting cognitive data.

III. Single unit fuzzy event cognitive computing based on goal

A. Single unit fuzzy event and cognition

Single unit fuzzy event was a kind of cognitive theory proposed by the paper[7], the fact that some cognitive units interacted and generated a new cognitive unit was defined as single unit fuzzy event, wrote $e=\left[x_{1}\left(a_{x 1}\right), x_{2}\left(a_{x 2}\right), \ldots \ldots, x_{n}\left(a_{x n}\right)\right] \rightarrow y\left(a_{y}\right)(n \geqq 2)$, and the single unit fuzzy event was processed as the cognition, the cognition was wrote $c=f\left[x_{1}\left(b_{x 1}\right), x_{2}\left(b_{x 2}\right), \ldots \ldots, x_{n}\left(b_{x n}\right)\right] / m \rightarrow \overline{y(1)}$.

\section{B. Preprocessing of the cognitive data}

Definition 1 work and method

A work is the single unit fuzzy event that all inputting cognitive units are empty, writes $w=[\varnothing] \rightarrow y\left(a_{y}\right), \quad \varnothing \quad$ means that all inputting cognitive units are empty, so the single unit fuzzy event doesn't occur. If the $\varnothing$ is replaced by a method, the single unit fuzzy event will occur, so the method is all inputting cognitive units of the single unit fuzzy event, writes $m \propto$ $w=x_{1}\left(a_{x 1}\right), x_{2}\left(a_{x 2}\right), \ldots \ldots, x_{n}\left(a_{x n}\right)$. Work is proposed from the outside of cognition, the finished work is that the method has been calculated by reforming cognitive sub-computing, the unfinished work is that the method hasn't been calculated by reforming cognitive sub-computing.

Definition 2 problem

Problem is the unfinished work that stored in the problems pool and writes $p n_{\mathrm{i}} \propto[0] \rightarrow y\left(a_{y}\right), 0$ shows that the work is a unfinished work and stores in problems pool. Problems pool is a memory which storage capacity is finite, while it is full, the number that stores problems calls maximum capacity, the maximum capacity is fixed, as showed in figure 2.

\begin{tabular}{|c|c|c|}
\hline$y_{1}$ & $a_{y 1}$ & 0 \\
\hline$y_{2}$ & $a_{y 2}$ & 0 \\
\hline$\cdots \cdots$ & $\cdots \cdots$ & 0 \\
\hline$y_{m}$ & $a_{y m}$ & 0 \\
\hline
\end{tabular}

Fig.2 A problems pool

In figure 2, the problems pool is full and the maximum capacity is $m$, the number that the problems pool is able to store problems arrays from 1 to $m$.

\section{Definition 3 goal}

Goal is the mapping of the problems and cognitions at a certain moment, the problems were stored in the problem pool and wrote $p n_{1}, p n_{2}, \ldots . . p n_{\mathrm{m}}$, the cognitions were stored in memory bank and wrote $c_{1}, c_{2}, \ldots \ldots c_{\mathrm{n}}$, so the goal was wrote $g=f\left[\left(p n_{1}\right),\left(p n_{2}\right), \ldots \ldots\left(p n_{\mathrm{m}}\right)\right] \cup f\left[\left(c_{1}\right)\right.$, $\left.\left(c_{2}\right), \ldots \ldots\left(c_{\mathrm{n}}\right)\right]$. If one single unit fuzzy event or an inputting cognition correlates the problem stored in the problem pool or correlates the cognition stored in memory bank, the single unit fuzzy event or the inputting cognition must correlate the goal.

\section{1) Selecting cognitive sub-computing}

When one single unit fuzzy event occurs or an inputting cognition is inputted, selecting cognitive sub-computing works, it measures whether the single unit fuzzy event or the inputting cognition correlates the goal. The algorithm of selecting cognitive sub-computing is expressed as:

$g=f\left[\left(p n_{1}\right), \quad\left(p n_{2}\right), \ldots \ldots\left(p n_{m}\right)\right] \quad \cup \quad f\left[\left(c_{1}\right)\right.$, $\left.\left(c_{2}\right), \ldots \ldots\left(c_{\mathrm{n}}\right)\right]$ and $\left(e\right.$ or $\left.c_{\mathrm{in}}\right)$;

if $\left(e\right.$ or $\left.c_{\text {in }}\right) \propto\left(p n_{i}\right.$ or $\left.c_{i}\right)$;

then( $e$ processed as $c$, or $c_{\text {in }}$ processed as $c$ )and ( $c$ is stored in memory bank); deleting cognitive sub-computing; else exit; 


\section{2) Rejecting cognitive sub-computing}

A work is provided while the problems pool is full, then rejecting cognitive sub-computing works, the work is be rejected and cognition doesn't work. The algorithm of deleting cognitive sub-computing is expressed as:

$g=f\left[\left(p n_{1}\right), \quad\left(p n_{2}\right), \ldots\left(p n_{i-1}\right),\left(p n_{i+1}\right), \ldots\left(p n_{\mathrm{m}}\right)\right] \cup$ $f\left[\left(c_{1}\right),\left(c_{2}\right), \ldots \ldots\left(c_{\mathrm{n}}\right)\right]$ and (PP is full);

$\left(t_{i}\right)$;

$g=f\left[\left(p n_{1}\right), \quad\left(p n_{2}\right), \ldots\left(p n_{i-1}\right),\left(p n_{i+1}\right), \ldots\left(p n_{\mathrm{m}}\right)\right] \cup$ $f\left[\left(c_{1}\right),\left(c_{2}\right), \ldots \ldots\left(c_{\mathrm{n}}\right)\right]$

\section{Single unit fuzzy event cognitive computing based on goal}

The structure of single unit fuzzy event cognitive computing based on goal is showed in figure 3 , except preprocessing of the cognitive data, it consists of understanding cognitive sub-computing, learning cognitive sub-computing, reforming cognitive sub-computing and deleting cognitive sub-computing.

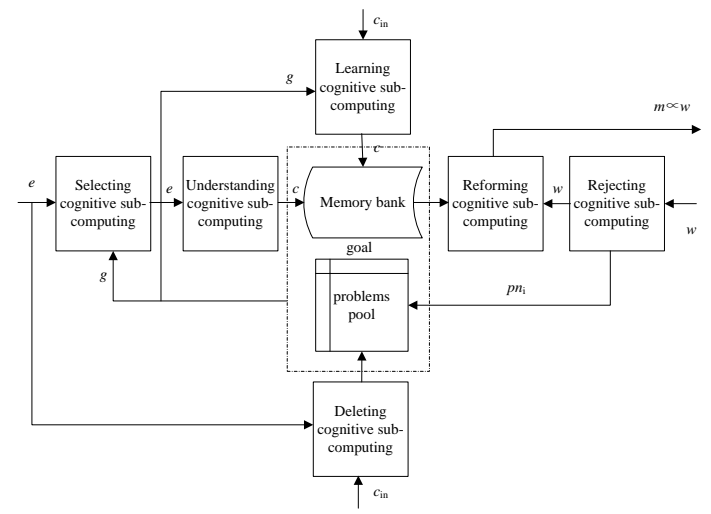

Fig.3 The structure of single unit fuzzy event cognitive computing based on goal

1) Understanding cognitive sub-computing

When understanding cognitive sub-computing works, the single unit fuzzy event is processed as the cognition by understanding cognitive sub-computing, the algorithm of understanding cognitive sub-computing is expressed as:

$$
e=\left[x_{1}\left(a_{x 1}\right), x_{2}\left(a_{x 2}\right), \ldots \ldots, x_{n}\left(a_{x n}\right)\right] \rightarrow y\left(a_{y}\right) ;
$$

$c_{i} \propto e$

$c_{i}=f\left[x_{1}\left(b_{x 1}{ }^{\prime}\right), x_{2}\left(b_{x 2}{ }^{\prime}\right), \ldots \ldots, x_{n}\left(b_{x n}{ }^{\prime}\right)\right] /(m-$

$1) \overline{y(1)}$;

$$
s a=\sum_{i=1}^{n} a x_{i}
$$

$b_{x i}=b_{x i}^{\prime}+\left(\frac{a x_{i}}{s a}-b_{x i}{ }^{\prime}\right) \times \frac{a_{y}}{m^{2}} ;$

$c=f\left[x_{1}\left(b_{x 1}{ }^{\prime}+\left(\frac{a x_{1}}{s a}-b_{x 1}{ }^{\prime}\right) \times \frac{a_{y}}{m^{2}}\right), x_{2}\left(b_{x 2}{ }^{\prime}+\left(\frac{a x_{2}}{s a}-\right.\right.\right.$

$\left.\left.b_{x 2}{ }^{\prime}\right) \times \frac{a_{v}}{m^{2}}\right), \ldots \ldots, \quad x_{n}\left(b_{x n}{ }^{\prime}+\left(\frac{a x_{n}}{s a}-b_{x n}{ }^{\prime}\right) \times \frac{a_{v}}{m^{2}}\right.$

)$] / m \rightarrow \overline{y(1)}$

\section{2) Learning cognitive sub-computing}

When learning cognitive sub-computing works, the inputting cognition is processed as the cognition by learning cognitive sub-computing, the algorithm of learning cognitive sub-computing is expressed as:

$$
\begin{aligned}
& c_{\text {in }}=f\left[x_{1}\left(b_{x 1}\right), x_{2}\left(b_{x 2}{ }^{\prime}\right), \ldots \ldots, x_{n}\left(b_{x n}{ }^{\prime}\right)\right] / 0 \rightarrow \overline{y(1)} ; \\
& c=f\left[x_{1}\left(b_{x 1}\right), x_{2}\left(b_{x 2}\right), \ldots \ldots, x_{n}\left(b_{x n}\right)\right] / m \rightarrow \overline{y(1)} ; \\
& c_{\text {in }} \propto c ; \\
& c=f\left[x_{1}\left(b_{x 1}\right), x_{2}\left(b_{x 2}{ }^{\prime}\right), \ldots \ldots, x_{n}\left(b_{x n}{ }^{\prime}\right)\right] / 0 \rightarrow \overline{y(1)} ; \\
& \text { 3) Reforming } \\
& \text { sub-computing }
\end{aligned}
$$

\section{3) Reforming cognitive sub-computing}

A work is provided while the problems pool isn't full, reforming cognitive sub-computing works, the work writes $w=[\varnothing] \rightarrow y\left(a_{y}\right)$, $\varnothing$ means that the cognitive unit of single unit fuzzy event is empty, so the single unit fuzzy event doesn't occur, the method of the work is the cognitive unit of single unit fuzzy event. The algorithm of reforming cognitive sub-computing is expressed as:

$$
\begin{aligned}
& w=[\varnothing] \rightarrow y\left(a_{y}\right) ; \\
& w \propto c_{C} ; \\
& c=f\left[x_{1}\left(b_{x 1}\right), x_{2}\left(b_{x 2}\right), \ldots \ldots, x_{n}\left(b_{x n}\right)\right] / m \rightarrow \overline{y(1)} ; \\
& m \propto{ }_{w}=x_{1}\left(a_{x 1}\right), x_{2}\left(a_{x 2}\right), \ldots \ldots, x_{n}\left(a_{x n}\right) ;
\end{aligned}
$$


$a_{x i}=\mathrm{k} b_{x i}$ and $\mathrm{k} b_{x i} \leq 1 \quad(\mathrm{k}$ is worksite constant).

4) Deleting cognitive sub-computing

When deleting cognitive sub-computing works, it deletes the problem which correlates the single unit fuzzy event from the problems pool. The algorithm of deleting cognitive sub-computing is expressed as:

$g=f l\left(p n_{1}\right), \quad\left(p n_{2}\right), \ldots, \quad\left(p n_{i-1}\right), \quad\left(p n_{i}\right)$, $\left.\left(p n_{i+1}\right), \ldots\left(p n_{\mathrm{m}}\right)\right] \cup f\left[\left(c_{1}\right),\left(c_{2}\right), \ldots \ldots\left(c_{\mathrm{n}}\right)\right]$ and $(e$ or $\left.C_{\text {in }}\right)$;

if $\left(e\right.$ or $\left.c_{\text {in }}\right) \propto p n_{i}$;

then delete $p n_{i}$ from PP;

$g=f\left[\left(p n_{1}\right),\left(p n_{2}\right), \ldots\left(p n_{i-1}\right),\left(p n_{i+1}\right), \ldots\left(p n_{\mathrm{m}}\right)\right] \cup$

$f\left[\left(c_{1}\right),\left(c_{2}\right), \ldots \ldots\left(c_{\mathrm{n}}\right)\right]$;

else exit;

5) Memory bank

All the cognition is stored in the memory bank, as showed in figure 4. Memory bank is like a table, the top line stores the mark of the result unit $y$, the mark of the cognitive unit $x_{i}$ and the mark of the experience data $m$, the other lines store cognition, per line stores a set of cognition. In figure 4, the memory bank stores four sets of cognition, they are $c_{2}=$ $f\left[x_{1}(0.62), x_{4}(0.38)\right] / 3 \rightarrow \quad \overline{y_{2}(1)}$ ,$C_{4}=f\left[x_{1}(0.28), x_{3}(0.58), x_{4}(0.14)\right] \quad / 5 \rightarrow \overline{y_{4}(1)}$, $c_{1}=f\left[x_{1}(0.16)\right.$, $\left.x_{2}(0.14), x_{3}(0.48), x_{4}(0.22)\right] / 1 \rightarrow \overline{y_{1}(1)} \quad, \quad c_{3}=$ $f\left[x_{2}(0.49), x_{3}(0.44), x_{4}(0.07)\right] / 21 \rightarrow \overline{y_{3}(1)}$.

\begin{tabular}{|c|c|c|c|c|c|}
\hline$y$ & $x_{4}$ & $x_{1}$ & $x_{2}$ & $x_{3}$ & $m$ \\
\hline$\overline{y_{2}(1)}$ & 0.38 & 0.62 & & & 3 \\
\hline$\overline{y_{4}(1)}$ & 0.14 & 0.28 & & 0.58 & 5 \\
\hline$\overline{y_{1}(1)}$ & 0.22 & 0.16 & 0.14 & 0.48 & 1 \\
\hline$\overline{y_{3}(1)}$ & 0.07 & & 0.49 & 0.44 & 21 \\
\hline
\end{tabular}

Fig.4 The memory bank
The volume of the memory bank equals the amount of the cell storing data, but the volume of the memory bank is constant. As showed in figure 5 , there are three kinds of storing cognition in the same memory bank; the volume of the memory bank is 22. In figure 5a, the memory stores four sets of cognition, the amount of lines is 5 , the amount of columns is 5; In figure 5b, the memory stores three sets of cognition, the amount of lines is 4 , the amount of columns is 6 ; In figure $5 c$, the memory stores two sets of cognition, the amount of lines is 3 , the amount of columns is 9 .
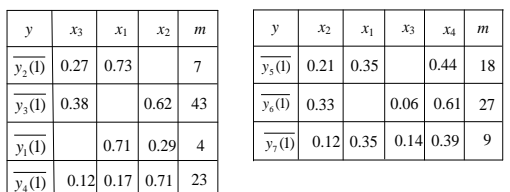

$\mathrm{a}$
\begin{tabular}{|c|c|c|c|c|c|c|c|c|}
\hline$y$ & $x_{6}$ & $x_{5}$ & $x_{2}$ & $x_{4}$ & $x_{3}$ & $x_{7}$ & $x_{1}$ & $m$ \\
\hline$\overline{y_{8}(1)}$ & 0.12 & 0.06 & 0.20 & 0.17 & 0.11 & 0.23 & 0.12 & 1 \\
\hline$\overline{y_{9}(1)}$ & 0.54 & & & & & 0.46 & & 43 \\
\hline
\end{tabular}

Fig.5 Three kinds of storing cognition in the same memory bank

\section{Single fuzzy unit events cognitive computing experiment and results}

\section{A. Cognitive Computation Performance Figure (CCPF)}

The performance of cognitive computing is measured in cognitive computation performance figure, the cognitive computation performance figure includes work achievement rate $\eta$, problem repeating rate $\sigma$, forgetting rate $\rho$, selecting rate $\varphi$ and rejecting rate $\psi$.

\section{B. The experimental samples 1) Experimental data}

a) Work-event-work flow

The work-event-work flow is written $f_{\text {wew }}=w \rightarrow e_{1} \rightarrow e_{2} \rightarrow \ldots \ldots \rightarrow e_{p} \rightarrow w$, the size of the work-event-work flow is the amount of all the fuzzy events and works, so the size of the $f_{\text {wew }}$ equals $p+2$.

b) Work-inputting cognition-work flow

The work-inputting cognition-work flow is 
written $f_{\text {wiw }}=w \rightarrow c_{\text {in } 1} \rightarrow c_{\text {in } 2} \rightarrow \ldots . . \rightarrow c_{\text {inq }} \rightarrow w$, the size of the work-inputting cognition-work flow is the amount of all the inputting cognition and works, so the size of the $f_{\text {wiw }}$ equals $q+2$.

\section{2) The experiment database}

The experiment database was composed of 136 work-event-work flows and 112 work-inputting cognition-work flows, the amount of flows simulated the cognition in social science was 73, the size of all flows arrayed from 7 to16, so the experiment database could simulated the cognition.

\section{3) Cognitive computing process (CCP)}

Figure 6 is a cognitive process example, it consists of two work-event-work flows and one work-inputting cognition-work flow, the two work-event-work flows are $f_{\text {wew } 1}$ and $f_{\text {wew2 } 2}$, the one work-inputting cognition-work flow is $f_{\text {wiw } 1}$, $f_{\text {wew } 1}=\quad w_{1} \rightarrow e_{1} \rightarrow e_{2} \rightarrow e_{3} \rightarrow w_{1}, \quad f_{\text {wew } 2}=$ $w_{2} \rightarrow e_{4} \rightarrow e_{5} \rightarrow e_{6} \rightarrow w_{2}, f_{\text {wiw } 1}=w_{3} \rightarrow c_{\text {in }} \rightarrow w_{3}$.

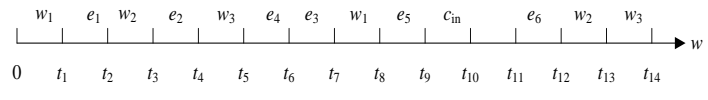

Fig.6 A cognitive computation process

\section{The cognitive computing experiment} and results

One cognitive computing process was consisted of $a$ work-event-work flows and $b$ work-inputting cognition-work flows. There were 128 cognitive computing process were created in the cognitive computing experiment, the sum which $a$ added $b$ equaled 22 in each cognitive computing process, the volume of the memory bank was 522 and the maximum capacity of the problems pool was 6 in the cognitive computing experiment. The memory bank and the problems pool were empty at 0 time in each cognitive computing process, the goals were created by the cognition and the problems which were automatically built by the single unit fuzzy event cognitive computing based on goal, the cognitive computation performance figures were calculated at the ended time of each cognitive computing process, the experimental results were shown at table 1.

TABLE I Experimental results of the cognitive

\begin{tabular}{llllllll}
\multicolumn{7}{c}{ computation (unit of CCPF \%) } \\
\hline & $a$ & $b$ & $\eta$ & $\sigma$ & $\psi$ & $\varphi$ & $\rho$ \\
\cline { 2 - 8 } CCP00 & 8 & 12 & 45.5 & 9.1 & 0 & 8.2 & 13.6 \\
CCP0 & 11 & 9 & 34.1 & 27.3 & 4.5 & 7.2 & 36.4 \\
CCP00 & 15 & 5 & 47.7 & 4.5 & 0 & 7.7 & 9.1 \\
$\ldots$ & & & & & & & \\
CCP05 & 13 & 7 & 50.0 & 0 & 0 & 8.9 & 0 \\
CCP05 & 4 & 16 & 47.7 & 4.5 & 0 & 8.7 & 4.5 \\
CCP05 & 12 & 8 & 45.5 & 9.1 & 0 & 8.0 & 18.2 \\
$\ldots$ & & & & & & & \\
CCP08 & 11 & 9 & 43.2 & 13.6 & 0 & 8.6 & 22.7 \\
CCP08 & 7 & 13 & 50.0 & 0 & 0 & 9.1 & 0 \\
CCP08 & 10 & 10 & 31.8 & 27.3 & 9.1 & 6.7 & 40.9 \\
$\ldots$ & & & & & & & \\
CCP12 & 2 & 18 & 50.0 & 0 & 0 & 8.9 & 4.5 \\
CCP12 & 14 & 6 & 45. & 9.1 & 0 & 8.5 & 9.1 \\
CCP12 & 5 & 15 & 47. & 4.5 & 0 & 8.7 & 4.5 \\
\hline
\end{tabular}

The experimental results showed that all selecting rate were ideal and stable, they were in [6.6\%, 9.2\%], so cognitive data preprocessing worked well in the single unit fuzzy event cognitive computing based on goal; The experimental results showed that the work achievement rate $\eta$, the problem repeating rate $\sigma$ and the rejected rate $\psi$ were ideal while the forgetting rate $\rho$ was very small in all 128 cognitive computing process, and the work achievement rate $\eta$, the problem repeating rate $\sigma$ and the rejected rate $\psi$ were bad while the forgetting rate was very big in all 128 cognitive computing process ,so the single unit fuzzy event cognitive computing based on goal worked well.

\section{Conclusion}

The single unit fuzzy event cognitive computing based on goal corrects the shortage of the existing cognitive computing, it's cognitive computation performance figure can measure the performance of the single unit fuzzy event cognitive computing based on goal, the rules that the cognitive computation performance figure varies are accord with cognition, so cognition is able to be expressed with the single unit fuzzy event cognitive computing based on goal. 


\section{REFERENCES}

[1]. Ioannidis J, Munafò M R, Fusar-Poli $P$, et al. Publication and other reporting biases in cognitive sciences: detection, prevalence, and prevention[J]. Trends in cognitive sciences, 2014, 18(5) , pp. 235-241.

[2]. Savitha R, Suresh S, Kim H J. A meta-cognitive learning algorithm for an extreme learning

machine classifier[J]. Cognitive Computation, 2013, pp. 1-11.

[3]. Laird John E, Newell Allen, Rosenbloom Paul S. SOAR: An Architecture for General Intelligence .Artificial Intelligence, 1987, 33(1) , pp.1-64.

[4]. Anderson John R, Bothell Daniel, Byrne Michael $\mathrm{D}$, et al. An Integrated Theory of the Mind. Psychological Review, 2004, 111(4) , pp.1036-1060.

[5]. José L Subirats,José M Jerez, Iván Gómez, et al. Multiclass Pattern Recognition Extension for the New C-Mantec Constructive Neural Network Algorithm. Cognitive Computation,2010, 2(4) , pp.285-290

[6]. Xu L, Wang Y, Hong Y, et al. Brain Scale-free Properties in Awake Rest and NREM Sleep: A Simultaneous EEG/fMRI Study[J]. Brain Topography, 2015, 28(2):292-304.

[7]. FENG Kang. Brain storage model based on single fuzzy cognitive unit event[J]. Computer Engineering and Science, 2013,35(7), pp.31-38. 